\title{
Erratum to: Evaluation of multiplex PCR in first episodes of febrile neutropenia as a tool to improve early yeast diagnosis in leukemic/preleukemic patients
}

\author{
H. M. Teixeira • C. Matias • J. J. F. Magalhães • \\ J. M. A. Lyra • H. R. L. Melo • M. B. Jucá • \\ C. A. A. Brito $\cdot$ N. Lucena-Silva $\cdot$ V. Magalhães
}

Published online: 15 July 2014

(C) Springer-Verlag Berlin Heidelberg 2014

\section{Erratum to: Support Care Cancer}

DOI: $10.1007 / \mathbf{s 0 0 5 2 0 - 0 1 4 - 2 3 0 5 - 1}$

The original version of this article unfortunately contained errors. The author written as L.Silva is wrong. The actual name is N.Lucena-Silva. And the author written as J.F.F.Magalhães is also wrong. The actual name is J.J.F.Magalhães

The correct order of authors should be:

H.M. Teixeira, C. Matias, J.J.F. Magalhães, J.M.A. Lyra, H.R.L. Melo, M.B. Jucá, C.A.A. Brito, N. Lucena-Silva, V. Magalhães.

The online version of the original article can be found at http://dx.doi.org/ 10.1007/s00520-014-2305-1.

H. M. Teixeira $(\varangle) \cdot$ C. Matias $\cdot$ H. R. L. Melo • M. B. Jucá $\cdot$

V. Magalhães

Tropical Medicine Department, Federal University of Pernambuco,

Recife, Brazil

e-mail: hebertonmt@gmail.com

\section{J. J. F. Magalhães • J. M. A. Lyra • N. Lucena-Silva}

Aggeu Magalhães Research Center, Oswaldo Cruz Foundation,

Institute of Integral Medicine Prof. Fernando Figueira, Recife, Brazil

C. A. A. Brito

Clinical Medicine Department, Federal University of Pernambuco,

Recife, Brazil 